\title{
Propuesta de puntos de corte para diferentes indicadores antropométricos en la predicción de la incontinencia urinaria en mujeres
}

\author{
Cinara Sacomori ${ }^{1}$, Carla Rodrigues Vinter ${ }^{1}$, Fabiana Flores Sperandio ${ }^{1}$, Érico Felden \\ Pereira ${ }^{1}$, Fernando Luiz Cardoso. ${ }^{1}$ \\ ${ }^{1}$ Universidad del Estado de Santa Catarina - UDESC, SC, Centro de Ciencias de la Salud y del Deporte - CEFID. Brasil.
}

\section{RESUMEN}

Introducción: Los episodios de pérdidas urinarias son causas de vergüenza social, disfunción sexual y bajo desempeño profesional. Objetivos: Identificar la sensibilidad y especificidad, y proponer puntos de corte de diferentes indicadores antropométricos para la predicción en mujeres de la incontinencia urinaria (IU). Métodos: Fueron evaluadas 500 mujeres de la Gran Florianópolis, SC, Brasil, de un amplio rango etario, a partir del cuestionario ICIQ - SF para incontinencia urinaria. Fueron analizados los siguientes indicadores antropométricos: Circunferencia de Cintura (CC), Índice de Masa Corporal (IMC), Índice Cintura/Cadera (ICC), Razón Cintura/Estatura (RCE), Índice de Adiposidad Corporal (IAC) e Índice de Conicidad (Índice C). Para el análisis estadístico de los datos, fueron utilizadas pruebas de asociación y cálculos de sensibilidad y especificidad por medio de curvas ROC. Resultados: La prevalencia de IU fue $35 \%$. Se observaron correlaciones significativas entre los indicadores antropométricos y frecuencia e intensidad de pérdidas urinarias. La RCE fue el indicador antropométrico con mayor área sobre la curva ROC $(0,618)$. La CC fue el indicador antropométrico con mayor sensibilidad y el Índice $C$, aquel con la mayor especificidad para la predicción de la IU. Mujeres con CC con $78,5 \mathrm{~cm}$ o más presentaron 1,24 vez más prevalencia de IU $(p<0,05)$. Mujeres con Índice $C$ de 1,23 puntos o más presentaron 1,23 vez más prevalencia de IU $(p<0,05)$. Conclusiones: Los indicadores antropométricos, especialmente aquellos que llevan en consideración la CC, presentaron asociaciones significativas y pueden ser usados como indicativos de IU.

PALABRAS CLAVE: Sobrepeso, antropometría, incontinencia urinaria, actividad física

\section{SUMMARY}

Background: The episodes of urinary incontinence may cause social embarrassment, sexual dysfunction and diminished work performance. Objectives: To identify the sensitivity, specificity and to propose cutoff points of different anthropometric indicators for the prediction of urinary incontinence (UI) in women. Methods: 500 women from Florianópolis, SC, Brazil, from a wide age range, were assessed using the Internatinal Consultation on Incontinence Questionnaire (ICIQ - SF). The anthropometric indexes analyzed were: Waist Circumference (WC), Body Mass Index (BMI), Waist / Hip Ratio (WHR), Waist / Height Ratio (WHeR), Body Adiposity Index (IAC) and Conicity Index (Index C). For statistical analysis, association tests and calculations of sensitivity and specificity by ROC curves were used. Results: The prevalence of UI was $35 \%$. There were significant correlations between anthropometric indicators and frequency and intensity of UI. The WHeR was the index with the largest area under the ROC curve (0.618). The WC was the most sensitive index and the index $C$ had the highest specificity. Women with WC with $78.50 \mathrm{~cm}$ or more exhibited 1.24 increased prevalence of $U I(p<0.05)$. Those women with index $C$ of 1.23 or more showed 1.23 
greater prevalence of UI $(p<0.05)$. Conclusions: Anthropometric indicators, especially those which take into account the WC, showed significant associations and can be used as predictors of UI.

\section{KEY WORDS: Overweight, anthropometry, urinary incontinence, physical activity}

\section{INTRODUCCIÓN}

De acuerdo con la definición propuesta por la Sociedad Internacional de Continencia, la incontinencia urinaria (IU) es considerada cualquier pérdida involuntaria de orina que cause problemas sociales o de higiene, estando relacionada al compromiso de la calidad de vida. La prevalencia de IU ha sido estimada de 10 a $50 \%$ en la población mundial $(1,2)$.

Varios factores de riesgo pueden contribuir al aumento de la prevalencia de IU, siendo estos: edad, paridad, enfermedades crónicas como diabetes, hipertensión, depresión, constipación, menopausia, cirugías ginecológicas previas, lesiones en el esfínter, tabaquismo y consumo de alcohol (3-6).

En medio de los múltiples factores de riesgo, la obesidad se ha destacado como un fuerte predictor de IU. La hipótesis explicativa para esto, es que el exceso de peso causa aumento de la presión intraabdominal con el consecuente aumento de la presión y movilidad vesical (3-9).

En algunos estudios se ha observado la relación entre el problema de exceso de peso corporal y episodios de pérdidas de orina, así como el hecho de que la reducción del peso corporal, obtenida por medio de dietas o incluso por cirugía, resulte en la reducción de la frecuencia de pérdida de orina. La gravedad de la incontinencia, además de ser más frecuente en mujeres obesas parece estar influenciada por el tiempo de duración de la obesidad (8,10-13).

A pesar de las asociaciones observadas entre la obesidad y la incontinencia urinaria, no está claro cuál indicador antropométrico sería el mejor indicador para ser utilizado en los estudios con IU en la práctica clínica, a la vez que los estudios disponibles evalúan por separado el índice de masa corporal o la circunferencia de la cintura. En el caso de otras enfermedades, como la diabetes y problemas cardiacos, los valores de referencia para estos indicadores antropométricos se investigan constantemente (14). Por lo tanto, el objetivo de este estudio fue identificar la sensibilidad y la especificidad y proponer puntos de corte de los diferentes indicadores antropométricos para la predicción de la incontinencia urinaria en mujeres.

\section{PACIENTES Y MÉTODO}

La población consta de aproximadamente 4200 mujeres. Estas fueron atendidas en la Red Femenina de Combate al Cáncer en la Gran Florianópolis, SC, Brasil, en el período comprendido entre agosto de 2011 y junio de 2012. Participaron de este estudio 500 mujeres con más de 18 años que realizaron exámenes de rutina para rastreo del cáncer de cuello uterino en las tardes de los martes, jueves y viernes, sin agendamiento previo. El estudio fue aprobado por el comité de ética en investigación de la Universidad del Estado de Santa Catarina (n.15/2011).

Instrumentos. Se utilizó el International Consultation on Incontinence Questionnaire - Short Form (ICIQ-SF), que está compuesto de cuatro ítems que investigan la frecuencia de pérdidas de orina (nunca, una vez por semana o menos, dos o tres veces a la semana, una vez al día, muchas veces al día, todo el tiempo), cantidad de pérdida de orina (ninguna, pequeña, media o grande), además de su impacto en la calidad de vida (escala análogo visual de 0 a 10), y un conjunto de ocho sub-ítems relacionados a las situaciones en que ocurrían las pérdidas. El puntaje del ICIQ-SF es obtenido sumándose las puntuaciones de los ítems anteriormente descritos, con variación de 0 a 21. Este cuestionario fue elaborado por Avery y cols (15) validado para el idioma portugués (16) y mostró ser una técnica no invasiva para identificar la IU con satisfactoria precisión, comparándolo con el patrón de oro, el test urodinámico (17).

Para obtención de las medidas antropométricas, se utilizó una balanza Inner Scan del modelo BC - 533/ Marca Tanita, una cinta métrica fijada en la pared para medición de la estatura, y una cinta métrica de la marca ISP para medición de las circunferencias de la cintura y de las caderas. La masa corporal fue obtenida en una balanza de plataforma nivelada y las mujeres fueron posicionadas en ella con ropas ligeras y descalzas. La estatura se medió con la mujer en postura erecta, con los brazos al lado del cuerpo y las manos hacia las piernas, pies descalzos, talones junto a la pared y el peso del cuerpo igualmente distribuido en ambos pies. En seguida, la mujer era instruida a mirar el horizonte 
e inspirar profundamente sosteniendo la respiración mientras la medición era efectuada. Para evaluación de las circunferencias de la cintura y caderas, la cinta métrica se colocó en paralelo al piso y sin apretar la piel o comprimir tejidos subcutáneos, la medida era obtenida luego debajo de las últimas costillas en la región de menor circunferencia.

La circunferencia de las caderas fue obtenida en la región de mayor circunferencia. El índice de masa/corporal (IMC) fue calculado por la fórmula [peso/estatura ${ }^{2}$ ]; el índice cintura/cadera (ICC) por la división matemática entre circunferencia de cintura (CC) y circunferencia de caderas, y la relación cintura/estatura, por la razón entre CC y estatura (cm). Para el Índice de conicidad, se consideró la siguiente fórmula: \{́lndice $\mathrm{C}=\mathrm{CC} / 0,109^{\star} \sqrt{ }$ [peso corporal $(\mathrm{kg}) /$ estatura $(\mathrm{m})]\}$. El índice de adiposidad corporal fue evaluado con la fórmula: $\{\mathrm{IAC}=$ circunferencia de la cadera $(\mathrm{cm}) /[$ (estatura $\left.\left.(m)^{1,5}\right]-18\right\}$.

Los datos sociodemográficos analizados fueron edad, escolaridad, estado marital, etnia, percepción del estado de salud y práctica de actividad física en el ocio.

Procedimientos. De inicio se proveyeron informaciones sobre los procedimientos de investigación por medio de instrucciones verbales. Si las participantes estaban de acuerdo, firmaban el término de consentimiento libre informado. En segui$\mathrm{da}$, las mujeres respondieron a los cuestionarios en forma de entrevista, debido al bajo nivel de escolaridad de muchas participantes. Finalmente, las mujeres fueron sometidas a las medidas físicas: masa corporal, estatura y circunferencias.

Análisis de los datos. Los datos fueron tabulados en el programa SPSS versión 17 y analizados con recursos de estadística descriptiva e inferencial. Para el análisis descriptivo, fueron usadas medidas de frecuencia (absoluta y relativa), tendencia central (media, mediana) y de dispersión (desviación estándar). Los datos no presentaron distribución normal, aún después de tratar de normalizarlos, por lo que se utilizaron los tests de Correlación de Spearman, Chi Cuadrado y U de Mann Whitney. Fue utilizada la curva receiver operating characteristic $(\mathrm{ROC})$ para estimar la capacidad predictiva de los indicadores antropométricos (masa corporal, CC, IMC, RCC, RCE, IAC e Índice C), tratando de evaluar el mejor punto de corte para predicción de IU. Además, fueron calculadas las razones de prevalencia considerando los puntos de corte para los indicadores antropométricos por medio de la regresión de Poisson. Fue adoptado un intervalo de confianza de $95 \%(p<0,05)$.

\section{RESULTADOS}

Caracterización de las participantes. La prevalencia de IU fue de $35 \%$, con predominio de pérdidas en pequeña cantidad de orina $(27,2 \%)$ y con frecuencia de una vez a la semana o menos $(20,2 \%)$ (Tabla I). La media de edad de las mujeres fue 40,9 $\pm 13,1$ años. Las mujeres incontinentes presentaron más edad que las continentes $(U=11261 ; p<0,001)$. Hubo diferencia significativa entre mujeres continentes e incontinentes en las proporciones relacionadas a la escolaridad y estado de salud, de modo que las mujeres incontinentes, en general, presentaron niveles inferiores de escolaridad y peor estado de salud. La proporción de mujeres activas e inactivas físicamente en el período de ocio, fueron semejantes entre mujeres continentes e incontinentes (Tabla I).

Medidas antropométricas en mujeres con $y$ sin pérdida de orina. Se observó que las mujeres incontinentes presentaron valores, en general, significativamente más altos que las continentes para los indicadores antropométricos investigados (Tabla II). Además, las características de pérdida urinaria (frecuencia, cantidad, interferencia en la vida diaria) presentaron correlación positiva con todos los indicadores antropométricos analizados (Tabla III).

El indicador antropométrico con más capacidad predictiva para la IU, con relación a sensibilidad, fue la circunferencia de la cintura (Tabla IV). A pesar de eso, todos los demás indicadores, con excepción del índice de adiposidad corporal, obtuvieron significancia estadística en la curva ROC. Con relación al Índice de conicidad, se comprobó un alto valor de especificidad, pero baja sensibilidad. La mayor área sobre la curva ROC para la IU fue observada en el Índice relación cintura/estatura.

\section{DISCUSIÓN}

Hubo una alta prevalencia de IU (35\%) en el grupo de mujeres que estaban realizando examen de rastreo de cáncer de cuello uterino. A pesar de la alta prevalencia, hubo un predominio de pérdidas en pequeña cantidad de orina y con baja frecuencia (una vez a la semana o menos). En este sentido, un meta-análisis verificó tasa de prevalencia que variaba de $10 \%$ a $50 \%$ en la población mundial, siendo el pico en la media edad (2).

Se observó que las mujeres incontinentes se auto-evaluaban con peor estado de salud comparadas con las continentes. Un peor estado de salud se relaciona con la presencia de comorbidades, tales como aquellas asociadas a IU: depresión, diabetes e hipertensión arterial (3-6). 


\section{Tabla I}

CARACTERÍSTICAS SOCIO-DEMOGRÁFICASY DE PERFIL DE PÉRDIDA URINARIA DE LAS MUJERES

\begin{tabular}{|c|c|c|c|c|c|c|c|}
\hline \multirow[t]{2}{*}{ Variables } & \multicolumn{2}{|c|}{$\begin{array}{l}\text { Todas } \\
(\mathrm{n}=500)\end{array}$} & \multicolumn{2}{|c|}{$\begin{array}{l}\text { Incontinentes } \\
(\mathrm{n}=175)\end{array}$} & \multicolumn{2}{|c|}{$\begin{array}{c}\text { Continentes } \\
(n=325)\end{array}$} & \multirow[t]{2}{*}{$x^{2}$} \\
\hline & $\mathrm{n}$ & $\%^{*}$ & $\mathrm{n}$ & $\%^{*}$ & $\mathrm{n}$ & $\%^{*}$ & \\
\hline \multicolumn{8}{|l|}{ Escolaridad } \\
\hline Enseñanza Fundamental & 222 & 44,8 & 102 & 58,6 & 120 & 37,3 & $X^{2}=22,1$ \\
\hline Enseñanza Media & 223 & 44,6 & 62 & 35,6 & 161 & 50 & $\mathrm{p}<0,001$ \\
\hline Enseñanza Superior & 51 & 10,2 & 10 & 5,7 & 41 & 12,7 & \\
\hline \multicolumn{8}{|l|}{ Estado Marital } \\
\hline Sin pareja & 134 & 26,8 & 46 & 26,3 & 88 & 27,1 & n.s \\
\hline Con pareja & 366 & 73,2 & 129 & 73,7 & 237 & 72,9 & \\
\hline \multicolumn{8}{|l|}{ Etnia } \\
\hline Caucasiana & 388 & 77,6 & 131 & 74,9 & 257 & 79,1 & n.s \\
\hline Negra/mulata/oriental & 112 & 22,4 & 44 & 25,1 & 68 & 20,9 & \\
\hline \multicolumn{8}{|l|}{ Percepción del Estado de Salud ${ }^{\star \star}$} \\
\hline Muy bueno/Bueno & 287 & 57,7 & 80 & 46,2 & 207 & 63,9 & $X^{2}=18,5$ \\
\hline Regular & 156 & 31,4 & 63 & 36,4 & 93 & 28,7 & $\mathrm{p}<0,001$ \\
\hline Malo/Muy malo & 54 & 10,9 & 30 & 17,3 & 24 & 7,4 & \\
\hline Activas física en tiempo libre & 254 & 50,8 & 85 & 48,6 & 169 & 52 & n.s \\
\hline \multicolumn{8}{|l|}{ Frecuencia de pérdidas de orina } \\
\hline Nunca & 325 & 65,0 & - & - & 325 & 100 & \\
\hline Una vez a la semana o menos & 101 & 20,2 & 101 & 57,7 & - & - & \\
\hline Dos veces a la semana o menos & 36 & 7,2 & 36 & 20,6 & - & - & \\
\hline Una vez aldía & 18 & 3,6 & 18 & 10,3 & - & - & \\
\hline Diversas veces al día & 18 & 3,6 & 18 & 10,3 & - & - & \\
\hline Todo el tiempo & 2 & 0,4 & 2 & 1,1 & - & - & \\
\hline \multicolumn{8}{|l|}{ Cantidad de pérdidas ${ }^{\star \star \star}$} \\
\hline Ninguna & 325 & 65,0 & - & - & 325 & 100 & \\
\hline Pequeña & 136 & 27,2 & 136 & 77,7 & - & - & \\
\hline Moderada & 21 & 4,2 & 21 & 12,0 & - & - & \\
\hline Grande & 17 & 3,4 & 17 & 9,7 & - & - & \\
\hline
\end{tabular}

*Debido a algunos missing cases fue utilizado el porcentaje válido; ** estado de salud evaluado con la pregunta: “¿Cómo consideras tu estado de salud? Muy bueno, bueno, regular, malo o muy malo"; ${ }^{\star \star \star}$ pequeña, moderada, grande cantidad = percepción de pequeño, medio o grande goteo de orina; $n . s=$ no significativo para $p<0,05$.

También se verificó en esta investigación, que las mujeres incontinentes eran más viejas que las continentes. Las alteraciones funcionales observadas con el envejecimiento reflejan cambios histológicos observados en el músculo estriado y tejidos conectivos de la uretra y vasos sanguíneos. Ocurre, con el pasar de los años, un proceso de reducción de las fibras tipo I y tipo II, además de peor respuesta a los estímulos eléctricos y electromiográficos. Se informado que el diámetro de las fibras musculares disminuye con la edad y ocurre una disminución de la relación de fibras musculares, mientras el tejido conectivo aumenta $(18,19)$.
Este estudio no encontró asociación entre la práctica de actividad física en tiempo libre y la IU. Eso puede ser atribuido al método utilizado para medición de la actividad física, el auto-relato, que no es muy preciso. Sin embargo, fue demostrado que la actividad física moderada puede proteger a las mujeres del riesgo de presentar IU. Además, otro estudio, comparó la función del piso pélvico de mujeres ancianas practicantes de actividad física con ancianas sedentarias, verificando que, las activas físicamente, tuvieron contracción muscular de las fibras lentas y rápidas más eficiente. Sin embargo, son necesarios más estudios sobre ese tópico $(7,20)$. 


\section{Tabla II \\ COMPARACIÓN DE LA EDADY MEDIDAS ANTROPOMÉTRICAS ENTRE MUJERES CONTINENTES E INCONTINENTES}

\begin{tabular}{|c|c|c|c|c|c|c|c|}
\hline \multirow[t]{2}{*}{ Variables } & \multicolumn{2}{|c|}{$\begin{array}{l}\text { Todas } \\
(n=500)\end{array}$} & \multicolumn{2}{|c|}{$\begin{array}{l}\text { Continentes } \\
\quad(n=325)\end{array}$} & \multicolumn{2}{|c|}{$\begin{array}{l}\text { Incontinentes } \\
\quad(n=175)\end{array}$} & \multirow[t]{2}{*}{$\begin{array}{c}\text { Test U de Mann } \\
\text { Whitney }\end{array}$} \\
\hline & $X( \pm D E)$ & Md & $X( \pm D E)$ & Md & $X( \pm D E)$ & Md & \\
\hline Edad (años) & $40,9(13,1)$ & 41,0 & $39,2(12,7)$ & 39,0 & $44,2(13,5)$ & 44,0 & $U=21045 ; p<0,001$ \\
\hline Masa Corporal (kg) & $66,0(12,0)$ & 64,0 & $64,3(11,1)$ & 62,0 & $68,9(13,1)$ & 67,0 & $U=22389 ; p<0,001$ \\
\hline $\begin{array}{l}\text { Circunferencia de Cintura } \\
\text { (cm) }\end{array}$ & $82,8(11,7)$ & 81,0 & $80,9(10,6)$ & 79,2 & $86,3(12,9)$ & 85,2 & $U=19321 ; p<0,001$ \\
\hline $\begin{array}{l}\text { Circunferencia de Cadera } \\
\text { (cm) }\end{array}$ & $101,7(9,3)$ & 101,0 & $100,5(8,8)$ & 100,0 & $103,5(10,0)$ & 103,5 & $U=20765 ; p<0,001$ \\
\hline $\begin{array}{l}\text { Índice de Masa Corporal } \\
\left(\mathrm{kg} / \mathrm{m}^{2}\right)\end{array}$ & $26,0(4,7)$ & 25,2 & $25,3(4,4)$ & 24,7 & $27,4(4,9)$ & 26,6 & $U=10738 ; p<0,001$ \\
\hline Índice Cintura/Cadera & $0,81(0,08)$ & 0,80 & $0,80(0,08)$ & 0,79 & $0,83(0,08)$ & 0,82 & $U=20485 ; p<0,001$ \\
\hline Razón Cintura/Estatura & $52,2(7,8)$ & 51,2 & $51,1(7,7)$ & 49,7 & $54,1(8,1)$ & 53,2 & $\mathrm{U}=4017 ; \mathrm{p}=0,004$ \\
\hline $\begin{array}{l}\text { Índice de Adiposidad } \\
\text { Corporal }\end{array}$ & $32,8(5,4)$ & 32,4 & $32,3(6,1)$ & 31,7 & $33,8(5,9)$ & 33,3 & $\mathrm{U}=4533 ; \mathrm{p}=0,091$ \\
\hline Índice de Conicidad & $1,18(0,09)$ & 1,17 & $1,17(0,1)$ & 1,2 & $1,19(0,1)$ & 1,2 & $U=4330 ; p=0,038$ \\
\hline
\end{tabular}

$\mathrm{X}=$ media, $\mathrm{Md}=$ mediana, $\mathrm{DE}=$ desviación estándar

Tabla III

CORRELACIONES ENTRE EDADY VARIABLES ANTROPOMÉTRICAS CON LAS CARACTERÍSTICAS DE INCONTINENCIA URINARIA

\begin{tabular}{|c|c|c|c|c|c|c|c|c|}
\hline \multirow[t]{2}{*}{ Variables } & \multicolumn{2}{|c|}{$\begin{array}{c}\text { Frecuencia } \\
\text { de IU }\end{array}$} & \multicolumn{2}{|c|}{$\begin{array}{l}\text { Cantidad de } \\
\text { pérdida de orina }\end{array}$} & \multicolumn{2}{|c|}{$\begin{array}{l}\text { Cuánto interfiere } \\
\text { en la vida diaria }\end{array}$} & \multicolumn{2}{|c|}{ ICIQ-SF } \\
\hline & $r$ & $\mathrm{p}$-valor* & $r$ & $\mathrm{p}$-valor* & $\mathrm{R}$ & $\mathrm{p}$-valor & r & p-valor \\
\hline Edad (años) & 0,203 & $<0,001$ & 0,193 & $<0,001$ & 0,151 & 0,001 & 0,192 & $<0,001$ \\
\hline Masa Corporal (kg) & 0,154 & 0,001 & 0,173 & $<0,001$ & 0,152 & 0,001 & 0,176 & $<0,001$ \\
\hline $\begin{array}{l}\text { Circunferencia de Cintura } \\
\text { (cm) }\end{array}$ & 0,231 & $<0,001$ & 0,235 & $<0,001$ & 0,239 & $<0,001$ & 0,248 & $<0,001$ \\
\hline $\begin{array}{l}\text { Circunferencia de Cadera } \\
(\mathrm{cm})\end{array}$ & 0,146 & 0,002 & 0,184 & $<0,001$ & 0,195 & $<0,001$ & 0,196 & $<0,001$ \\
\hline $\begin{array}{l}\text { Índice de Masa Corporal } \\
\left(\mathrm{kg} / \mathrm{m}^{2}\right)\end{array}$ & 0,189 & $<0,001$ & 0,221 & $<0,001$ & 0,201 & $<0,001$ & 0,233 & $<0,001$ \\
\hline Índice Cintura/Cadera & 0,193 & $<0,001$ & 0,186 & $<0,001$ & 0,163 & $<0,001$ & 0,188 & $<0,001$ \\
\hline Razón Cintura/Estatura & 0,235 & $<0,001$ & 0,250 & $<0,001$ & 0,251 & $<0,001$ & 0,261 & $<0,001$ \\
\hline $\begin{array}{l}\text { Índice de Adiposidad } \\
\text { Corporal }\end{array}$ & 0,161 & 0,001 & 0,191 & $<0,001$ & 0,213 & $<0,001$ & 0,206 & $<0,001$ \\
\hline Índice de Conicidad & 0,203 & $<0,001$ & 0,198 & $<0,001$ & 0,211 & $<0,001$ & 0,211 & $<0,001$ \\
\hline
\end{tabular}

*test de correlación de Spearman 


\section{Tabla IV \\ CAPACIDAD PREDICTIVA PARA INCONTINENCIA URINARIA, ÁREA DE LA CURVA ROC Y RAZONES DE PREVALENCIAS CON RELACIÓN A LOS INDICADORES ANTROPOMÉTRICOS}

\begin{tabular}{lcccccc}
\hline Indicadores & $\begin{array}{c}\text { Área } \\
\text { (IC 95\%) }\end{array}$ & p-valor & $\begin{array}{c}\text { Punto de } \\
\text { corte }\end{array}$ & $\begin{array}{c}\text { Sensibilidad } \\
(\text { IC 95\%) }\end{array}$ & $\begin{array}{c}\text { Especificidad } \\
(\text { IC 95\%) }\end{array}$ & $\begin{array}{c}\text { Razón de prevalencia } \\
(\text { IC 95\%) }\end{array}$ \\
\hline Masa Corporal & 0,574 & 0,067 & - & - & - & - \\
Índice de Masa Corporal & 0,606 & 0,008 & 25,82 & $59,3(47,8-70,0)$ & $61,2(52,5-69,3)$ & $1,21(1,06-1,37)$ \\
Circunferencia de Cintura & 0,610 & 0,006 & 78,50 & $72,2(60,9-81,7)$ & $50,7(42,0-59,4)$ & $1,24(1,09-1,40)$ \\
Razón Cintura/Cadera & 0,612 & 0,005 & 0,84 & $49,4(37,9-60,9)$ & $71,3(62,9-78,7)$ & $1,22(1,06-1,40)$ \\
Razón Cintura/Estatura & 0,618 & 0,003 & 51,72 & $59,0(47,3-70,0)$ & $63,7(55,0-71,8)$ & $1,23(1,08-1,40)$ \\
Índice de Adiposidad & 0,569 & 0,091 & - & - & - & - \\
Corporal & & & & & & $1,23(1,05-1,43)$ \\
Índice de Conicidad & 0,586 & 0,036 & 1,23 & $35,9(25,3-47,6)$ & $84,3(77,0-90,0)$ & 1 \\
\hline
\end{tabular}

*valores de Razón de prevalencia significativos para $\mathrm{p}<0,05$.

Se observó en este estudio que las mujeres con IU tenían valores de masa corporal, IMC, CC, RCC, IAC e Índice C significativamente mayor que las mujeres que no presentaban episodios de pérdidas de orina. El exceso de peso corporal es reconocido como un importante factor de riesgo para IU por contribuir en la alteración de la función de sustentación de los órganos pélvicos y de control miccional. Esos datos refuerzan la necesidad de implementación de estrategias de promoción de salud con enfoque en el estímulo de cambios de conductas sociales y ambientales relacionadas a la prevención de la obesidad (2-7).

Todos los indicadores antropométricos evaluados en este estudio, con excepción del índice de adiposidad corporal, presentaron significancia estadística en la curva ROC, pero ninguna área fue superior a 0,7, indicando limitaciones en la utilización de apenas un indicador para predicción de IU. Estudios con este enfoque son escasos, siendo observado solamente un estudio semejante con mujeres ancianas en Holanda que identificó un punto de corte de $95 \mathrm{~cm}$ para la circunferencia de la cintura como fuerte predictor de IU (21). Nuestro estudio estimó un punto de corte para circunferencia de cintura de $78,5 \mathrm{~cm}$ para mujeres con más de 18 años. Las correlaciones positivas obtenidas entre las variables relacionadas con la IU y todos los indicadores antropométricos, refuerzan la importancia de estos indicadores cuando se menciona la IU.

La asociación entre la presencia de IU y elevados valores de IMC y CC están bien descrita en la literatura. Mientras un estudio, con mujeres ancianas, identificó asociación del IMC y de la CC apenas con la IU de urgencia y mixta, otros estudios que incluyeron mujeres jóvenes (20/30 años o más) encontraron, también asociación de tales medidas con la IU de esfuerzo $(7,9,22,23,24)$.

En este estudio los indicadores antropométricos con más valor predictivo en relación a la incontinencia urinaria, fueron aquellos que consideraron la circunferencia de la cintura, esto por presentar más área sobre la curva ROC. Algunos autores defienden que la adiposidad abdominal, identificada por el aumento de la circunferencia de la cintura y de la razón cintura/cadera, está más asociada a la IU, al compararlo con otros índices más genéricos, como el IMC $(9,22)$. Así, las intervenciones para pérdida de peso se han mostrado efectivas en el tratamiento de la IU $(8,10,11)$. A partir de esto, también se sugiere que los estudios que evalúan factores de riesgo para IU, utilicen la medida antropométrica circunferencia de la cintura, además del IMC.

Se destaca como limitación del estudio no haber usado el test urodinámico para el diagnóstico de IU, ya que su elevado costo limita su utilización en estudios poblacionales. Se sugiere que sean conducidos estudios de base poblacional, investigando los factores descritos en este estudio, subsidiando la búsqueda de un diagnóstico más accesible de la incontinencia y la implementación de intervenciones profilácticas y adopción de orientaciones adecuadas y eficaces.

\section{CONCLUSIONES}

Los indicadores antropométricos son factores asociados con la ocurrencia de episodios de pérdidas urinarias en mujeres de la Gran Florianópolis, SC, Brasil. Además, esos indicadores 
también estuvieron asociados con el impacto de la IU en las actividades diarias de estas mujeres. En relación a la capacidad predictiva para IU, el indicador con más sensibilidad fue la circunferencia de la cintura; con más especificidad, el Índice de conicidad y con más área sobre la curva ROC, la razón cintura/estatura. Luego, los indicadores antropométricos, especialmente aquellos que consideraron la medida de la cintura en su cálculo, pueden ser utilizados como indicativos de IU, especialmente considerando los puntos de corte identificados.

\section{REFERENCIAS}

1. Abrams $\mathrm{P}$, Cardozo L, Fall M, Griffiths D, Rosier P, Ulmsten $U$, et al. The standardization of terminology in lower urinary tract function: Report from the standardization sub-committee of the International Continence Society. Neurourol Urodyn 2002;21(2):167-78.

2. Geelen J, Hunskaar S. The epidemiology of female urinary incontinence. Eur Clinics Obstet Gynecol 2005;1:3-11.

3. Higa R, Lopes $M$, Reis $M$. Fatores de risco para incontinência urinaria na mulher. Rev Esc Enferm USP 2008;42(1):187-92.

4. Hsieh C, Lee M, Kuo T, Hsu C, Chang S. Risk factors for urinary incontinence in Taiwanese women aged 20 - 59 years. Taiwan J Obstet Gynecol 2008;47(2):197202.

5. Melville J, Katon W, Delaney K, Newton K. Urinary Incontinence in US Women. Arch Inter Med 2005;165:537-42.

6. Fornell E, Wingren G, Kjølhede P. Factors associated with pelvic floor dysfunction with emphasis on urinary and fecal incontinence and genital prolapse: an epidemiological study. Acta Obst Gynecol Scand 2004;3(4):383-9.

7. Quu J, Lv L, Lin X, Long L, Zhu D, Xu R, et al. Body mass index, recreational physical activity and female urinary incontinence in Gansu, China. Eur J Obstet Gynecol Reprod Biol 2011;159:224-9.

8. Subak L, Whitcomb E, Shen H, Saxton J, Vittinghoff E, Brown J. Weight loss: a novel and effective treatment for urinary incontinence. J Urol 2005;174(1):190-5.

9. Han $\mathrm{M}$, Lee N, Park H. Abdominal obesity is associated with stress urinary incontinence in Korean women. Int Urogynecol J Pelvic Floor Dysfunct 2005;17(1):35-9.

10. Bump R, Sugerman H, Fantl J, McClish, D. Obesity and lower urinary tract function in women: effect of surgically induced weight loss. Am J Obstet Gynecol 1992;167(2):392-7.
11. Wing R, West D, Grady D, Creasman J, Richter $H$, Myers D, et al. Effect of weight loss on urinary incontinence in overweight and obese women: results at 12 and 18 months. J Urol 2010;184(3):1005-10.

12. Richter H, Kenton K, Huang L, Nygaard I, Kraus S, Whitcomb E, et al. The impact of obesity on urinary incontinence symptoms, severity, urodynamic characteristics and quality of life. J Urol 2010;183(2):622-8.

13. Pandey S, Bhattacharya S. Impact of obesity on gynecology. Womens Health 2010;6(1):107-17.

14. Thepsai U, Khanacharoen A, Manonai J, Chittacharoen A. Relationship between anthropometric measures of abdominal obesity and cardiovascular risk factors in women of different age groups. Int J Gynecol Obstet 2000;70(Sup 2):B79-B80.

15. Avery K, Donovan J, Peters T, Shaw C, Gotoh M, Abrams P. ICIQ: a brief and robust measure for evaluating the symptoms and impact of urinary incontinence. Neurourol Urodyn 2004;23:322-30.

16. Tamanini J, Dambros M, D'ancona C, Rodrigues Palma P, Netto Jr N. Validação para o português do "International Consultation on Incontinence Questionnaire - Short Form" (ISIQ-SF). Rev Saúde Pública 2004;38(3):438-44.

17. Timmermans L, Mélot C, Wespes E, Falez F. Analyse de la valeur du questionnaire ICIQ-sf et du test d'incontinence sur une heure pour le diagnostic d'incontinence urinaire objectivé par un bilan urodynamique. Pelvi-périnéologie 2011;6(3-4):162-5.

18. Chen G. Pelvic floor dysfunction in aging women. Taiwan J Obstet Gynecol 2007;46(4):374-8.

19. Corcos J, Mattison A. Female stress urinary incontinence. In: Bo K, Berghmans B, Morkved S, Kampen M. (Eds.). Evidence-based Physical Therapy for the Pelvic Floor London: Elsevier, 2007; pp 164-6.

20. Virtuoso J, Mazo G, Menezes E. Incontinencia urinaria e função muscular perineal em idosas praticantes e não - praticantes de atividade física regular. Rev Bras Fisioter 2011;15(4):310-7.

21. Heim N, Snijder M, Heymans M, Deeg D, Seidell J, Visser M. Optimal cutoff values for high-risk waist circumference in older adults based on related health outcomes. Am J Epidemiol 2011;174(4):479-89.

22. Krause M, Albert S, Elsangedy H, Krinski K, Goss F, Silva S. Urinary incontinence and waist circumference in older women. Age Ageing 2010;39(1):63-73.

23. Townsend M, Curhan G, Resnick N, Grodstein F. $\mathrm{BMI}$, waist circumference, and incident urinary incontinence in older women. Obesity 2008;6(4):881-6.

24. Parazzini F, Chiaffarino F, Lavezzari M, Giambanco V. Risk factors for stress, urge or mixed urinary incontinence in Italy. BJOG 2003;110(10):927-33. 